\title{
Monte Carlo simulations of transverse spin freezing in the three-dimensional frustrated Heisenberg model
}

\author{
J. R. Thomson, Hong Guo, D. H. Ryan, M. J. Zuckermann, and Martin Grant \\ Centre for the Physics of Materials, Department of Physics, McGill University, Rutherford Building, \\ 3600 University Street, Montréal, Québec H3A 2T8, Canada
}

\begin{abstract}
Monte Carlo simulations of the randomly frustrated three-dimensional Heisenberg model with a moderate fraction $(\leqslant 20 \%$ ) of antiferromagnetic bonds show evidence of two distinct ordering events. Below $T_{c}$ the spins align collinearly and exhibit ferromagnetic order, while below $T_{x y}$ the transverse components of the spins freeze leading to a noncollinear spin structure dominated by ferromagnetic correlations. The phase diagram and behavior of the calculated parameters agree qualitatively with those of experimental systems.
\end{abstract}

\section{INTRODUCTION}

Experimental studies of frustrated magnetic systems show that the presence of competing ferromagnetic (FM) and antiferromagnetic (AFM) interactions leads to a loss of collinearity in the ordered state and the formation of a spin glass. However, if the concentration of AFM bonds is small enough, it is possible to form a mixed exchange system with properties intermediate between the randomly frozen spin glass and the collinear ferromagnet. ${ }^{1}$ Such materials exhibit two magnetic transitions. The upper one (at $T_{c}$ ) is a continuation of the ferromagnetic transition and appears to mark the onset of long-range collinear magnetic order; however, there is a substantial transverse spin component present which precesses rapidly and time averages to zero. Below the second transition (at $T_{x y}$ ), the system ceases to be collinear as the transverse degrees of freedom order. $^{2}$

Theoretical support for a transverse spin freezing transition is unclear. The mean-field solution to the mixed exchange, three-dimensional (3D) Heisenberg model ${ }^{3}$ gives two magnetic transitions, first from the paramagnetic state to a collinear ferromagnet, then, at a lower temperature, to a mixed state where the spins acquire a significant transverse component. Recent simulation work on the $2 \mathrm{D} X Y$ model aimed at obtaining a physical picture of reentrant magnetic transitions was interpreted in terms of a spin canting transition at a temperature $T_{r}$, which was mediated by the "melting" of frustrated spins. ${ }^{4}$ They also report a transition at a higher temperature $T_{K}$ : As one decreases the temperature below $T_{K}$, the frustrated bonds make themselves felt, and the system begins to order in the transverse direction.

Spin canting is different from transverse spin freezing. On cooling through a spin canting transition, the magnetization $M$ decreases with respect to the total moment $S_{\text {rms }}$ (since the spins tilt away from their initially collinear alignment), while $S_{\text {rms }}$ itself only exhibits the normal increase due to the reduction in thermal fluctuations. Even at finite temperatures, where decreasing fluctuations may allow $M$ to increase through the spin canting transition, its temperature dependence exhibits a marked change in slope. The opposite occurs during transverse spin freezing: Here extra components order in the plane perpendicular to the $z$ axis, thus having no influence on $M$, but causing
$S_{\mathrm{rms}}$ to increase. Experimentally, there is a marked change in the behavior of $S_{r m s}$ at $T_{x y}$, often even a break in slope, but no such changes are seen in $M^{1,5}$

\section{THE MODEL}

We studied the simplest system which may be expected to exhibit transverse spin freezing: classical Heisenberg spins interacting on a simple cubic lattice according to the nearest-neighbor Hamiltonian

$$
\mathscr{H}=-\sum_{\langle i, j\rangle} J_{i j} \mathbf{S}_{i} \cdot \mathbf{S}_{j}-\mathbf{H} \cdot\left(\sum_{j} \mathbf{S}_{j}\right),
$$

with exchange strength $J_{i j}$ and spin lengths $\left|\mathbf{S}_{i}\right|=1$. The second term allows for an external magnetic field. For the ferromagnetic form of this model $\left(J_{i j}=+J\right)$ the ordering temperature $T_{c}$ is $T_{H}=1.44 J .^{6}$ Exchange frustration was introduced by randomly replacing a fraction $f$ of the bonds by $-J$ antiferromagnetic bonds. In order to investigate the behavior of the transverse spin components, the $z$ axis has to be specified. We have used two methods. One method is to define the $z$ axis externally by applying a small magnetic field $(|\mathbf{H}|=0.03 J)$. This also leads to shorter equilibration times by reducing the system's tendency to exploit global rotational degeneracy. Furthermore, most experimental observations of transverse spin freezing are made in this way. Alternatively, the $z$ axis may be identified with the direction of maximum spontaneous magnetization. The data presented below were obtained using the former method, but identical behavior is obtained in zero ficld for all valucs of $f$ studied here.

The results presented here are averages over at least 20 distinct bond configurations for each value of $f$ on $8^{3}$ lattices. Essentially, identical results have been obtained on $12^{3}$ and $8^{3}$ lattices. For each bond configuration the system was prepared well above $T_{c}$, then cooled to $T=0$ in 50 temperature steps using a Monte Carlo algorithm with Glauber dynamics. ${ }^{7}$ At each temperature, the system was allowed to equilibrate for 2000 Monte Carlo steps before local time averages were taken over 4000 Monte Carlo steps. The results were checked using 8000 Monte Carlo steps for time averaging with no appreciable difference.

We monitored two local time-averaged quantities, the first, a time average of the spin components at each site: 


$$
\mathbf{m}_{i}=\frac{1}{t} \sum_{t^{\prime}=0}^{t} \mathbf{S}_{i}\left(t^{\prime}\right),
$$

where $t=4000$ Monte Carlo steps and $i$ is the site index. The second was the average of the inner product of the transverse spin components at each site:

$$
m_{1 i}^{2}=\frac{1}{t} \sum_{t=0}^{t}\left[\mathbf{S}_{i}-\left(\mathbf{S}_{i} \cdot \hat{\mathbf{z}}\right) \hat{\mathbf{z}}\right]^{2} .
$$

These were then used to obtain the following global averages: the root-mean-square spin length,

$$
\left\langle S_{\mathrm{rms}}\right\rangle=\sqrt{\frac{1}{N} \sum_{i}\left(\mathbf{m}_{i} \cdot \mathbf{m}_{i}\right)},
$$

the bulk magnetization,

$$
M=\frac{1}{N}\left|\sum_{i} \mathbf{m}_{i}\right|,
$$

the root-mean-square transverse spin, which is a measure of the average component of the spins which lie perpendicular to the $z$ axis independent of direction or precession,

$$
Q_{\perp}=\frac{1}{N} \sum_{i} m_{1 i}^{2}
$$

and finally, the mean transverse spin length, which includes rotational averaging and measures the frozen transverse spin components,

$$
Q_{x y}=\frac{1}{N} \sum_{i}\left[\mathbf{m}_{i}-\left(\mathbf{m}_{i} \cdot \hat{\mathbf{z}}\right) \hat{\mathbf{z}}\right]^{2}
$$

\section{RESULTS AND DISCUSSION}

Figure 1 shows the temperature evolution of the various quantities defined above for a number of frustration levels. For $f=0$ all bonds are positive and the system is a Heisenberg ferromagnet below $T_{c}=T_{H}$. Since $S_{\mathrm{rms}}$ is essentially equal to $M$ at all $T<T_{c}$. the system is ordered collinearly. $Q_{1}$ decreases smoothly from $\frac{2}{3}$ above $T_{c}$, corresponding to an isotropic spin distribution, to zero at $T=0$, as expected for collinear order. $Q_{x y} \sim 0$ at all temperatures, indicating that there are no ordered transverse components.

New features appear for $f>0$. First, $Q_{\perp}$ (which measures the length of the transverse spins regardless of their direction in the $x-y$ plane) does not reach zero for any $\delta>0$, indicating that some degree of noncollinearity remains in the spin system even at $T=0$. More significantly, while the ordered transverse component monitored by $Q_{x y}$ is initially zero below $T_{c}$ as in the ferromagnetic case, it starts to grow at a well defined temperature $T_{x x y}$, below and separate from $T_{c}$, marking the onset of transverse spin freezing.

Second, the root-mean-square spin length $S_{\text {rms }}$, which followed the bulk magnetization $M$ on cooling from $T_{c}$ to $T_{x y}$, increases above $M$ and remains larger than it from $T_{x y}$ down to $T=0$. The increase of $S_{\mathrm{rms}}$ is a consequence of the transverse spin freezing. Above $T_{x y}$ when we examine a spin at a particular instant, it clearly possesses a
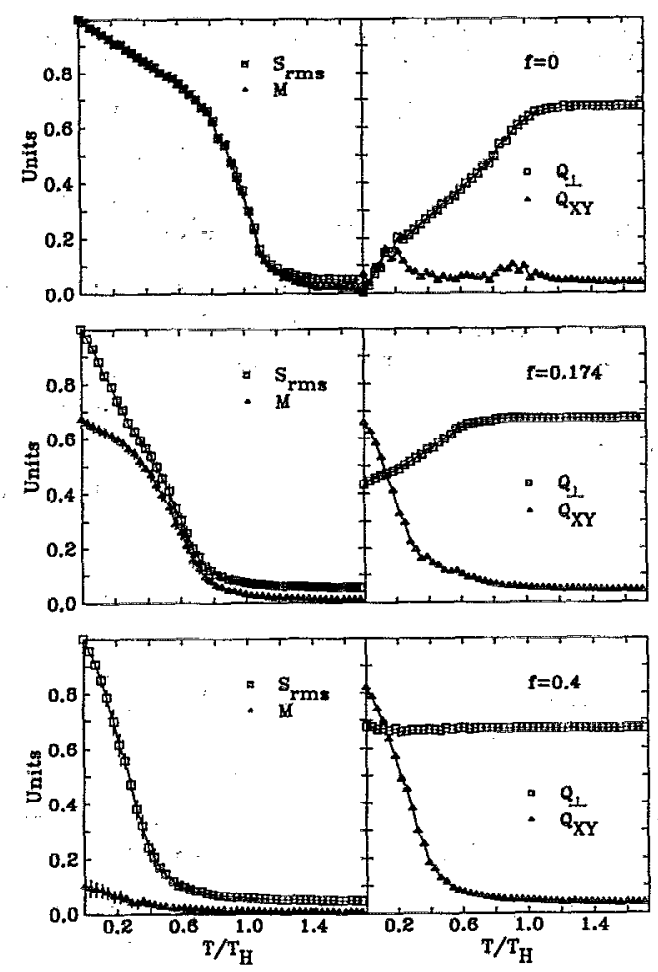

FIG. 1. Temperature dependence of longitudinal and transverse spin lengths for different levels of exchange frustration. The temperature scale is normalized to $T_{H}$. See text for definitions.

transverse component; however, the time avcrage of that component vanishes. Thus, $\mathbf{m}_{i}$ computed from (2) has only a $z$ component above $T_{x y}$, making $S_{\mathrm{rms}}$ and $M$ essentially equal and $Q_{x y}=0$. Below $T_{x y}$, the transverse spin components freeze at random, and so their site average cancels, leaving $M$ unaffected, but they do contribute to the sum given in (4) and increase $S_{\text {rms. }}$

The observation that $Q_{\perp}$ is large even above $T_{x y}$, but $Q_{x y}>0$ only occurs below $T_{x y}$ coupled with the increase of $S_{\text {rms }}$ over $M$ at the same temperature, is direct evidence of transverse spin freezing and is clearly incompatible with a spin canting model of this transition.

As $f$ is increased further, $T_{c}$ and $T_{x y}$ converge: $T_{c}$ falls as the average exchange strength declines, and $T_{x y}$ rises reflecting an increase in the exchange frustration. Finally, at $f \sim 0.25, T_{c}$ and $T_{x y}$ merge, and $Q_{x y}$ remains $\frac{2}{3}$ for all temperatures. This indicates that the spins are isotropically frozen in a spin-glass-like configuration. The calculated ordering behavior is very similar to that deduced from experiments; ${ }^{1,2,8}$ however, we caution that the lowtemperature behavior of a frustrated three-dimensional Heisenberg model is still an unresolved issue. ${ }^{8-10}$ If the lower critical dimension of this model is three or greater, there can be no spin-glass transition for $T>0$. In that case, both our results and the experiments mentioned above describe ordering on finite length scales within a presumably large correlation length.

The phase diagram given in Fig. 2 summarizes the frustration dependence of the various ordering events. The 


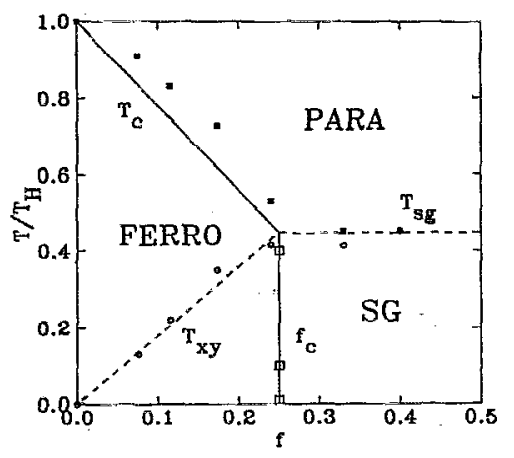

FIG. 2. Phase diagram for our model showing two magnetic transitions in the concentration region between $f=0$ and the spin glass at $f>0.25$. At $T_{c}$ collinear order sets in followed by the freezing of transverse spin components below $T_{x y} f_{c}$ marks the boundary between the ferromagnetic phase and spin glass. The temperature scale is normalized to $T_{H}$.

system is paramagnetic at high temperatures. Notwithstanding the reservations noted above, for $f>0.25$ the system enters a spin-glass phase on cooling through $T_{\mathrm{SG}}$. The vertical line at $f_{c}=0.25$ separates the spin-glass phase from the ferromagnetic phase and was determined from the frustration dependence of the fiuctuations in $M$, which corresponds to the static susceptibility. For $0<f<0.25$, the system goes through a transition at $T_{c}$ to a ferromagnetic phase. For a given $f$, this line marks the temperature where $S_{\mathrm{rms}}$ and $M$ deviate from zero. Below $T_{c}$, the order is dominated by strong ferromagnetic correlations, but there is a significant transverse spin component which time averages to zero. The dotted line $T_{x y}$ marks the temperature at which $Q_{x y}$ ceases to be zero and also corresponds to the separation point of $S_{\mathrm{rms}}$ and $M$. The system exhibits ferromagnetic order on both sides of the line, but below $T_{x y}$ the transverse spin components are frozen, whereas above it they are free and time average to zero. While we expect $T_{x y}$ to be a disorder line rather than a phase transition, further work is needed to fully determine the nature of the possible changes across this line.

\section{CONCLUSIONS}

Our Monte Carlo simulations are in good qualitative agreement with experimental observations. In particular, we found two magnetic transitions for moderate levels of frustration: collinear ordering at $T_{c}$ followed by transverse spin freezing at $T_{x y}$. We interpret the behavior at $T_{x y}$ in terms of a change in short-range order rather than a phase transition. Further work will examine the details of the low-temperature phase diagram more quantitatively.

\section{ACKNOWLEDGMENTS}

This work was supported by grants from the Natural Sciences and Engineering Research Council of Canada, and les Fonds pour la Formation de Chercheurs et l'Aide a' la Recherche de la Provincc du Québcc.

${ }^{1}$ R. A. Brand, V. Manns, and W. Keune, in Heidelberg Colloquium on Spin Glasses, Vol. 192 of Lecture Notes in Physics (Springer, Berlin, 1983), p. 79.

${ }^{2}$ D. H. Ryan, J. O. Ström-Olsen, R. Provencher, and M. Townsend, J. Appl. Phys. 64, 5787 (1988).

${ }^{3}$ M. Gabay and G. Toulouse, Phys. Rev. Lett. 47, 201 (1981).

${ }^{4}$ W. M. Saslow and G. Parker, Phys. Rev. Lett. 56, 1074 (1986).

${ }^{5}$ B. Huck and J. Hesse, J. Magn. Magn. Mater. 78, 247 (1989).

${ }^{\circ} \mathrm{C}$. Domb and M. S. Green, Phase Transitions and Critical Phenomena (Academic, London, 1974), Vol. 3.

${ }^{7}$ O. G. Mouritsen, Computer Studies of Phase Transitions and Critical Phenomena (Springer, Heidelberg, 1984).

${ }^{8}$ J. Souletie, Ann. Phys. Fr. 10, 69 (1985).

${ }^{9} \mathrm{~K}$. Binder and A. P. Young, Rev. Mod. Phys. 58, 801 (1086).

${ }^{10}$ A. Mauger, J. Villain, Y. Zhou, C. Rigaux, N. Bontemps, and J. Férré, Phys. Rev. B 41, 4587 (1990). 